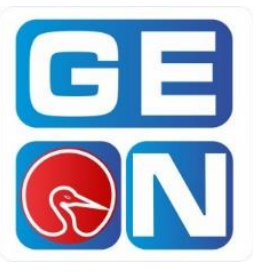

Revista GEON (Gestión, Organizaciones y Negocios.) ISSN: 2346-3910 en línea

revistageon@unillanos.edu.co

Universidad de los Llanos

Colombia

Valenzuela Salazar, Nemecio Lorenzoi; Buentello Martínez, Clara

Patricia ${ }^{\text {ii }}$; Gomez, Lilia Alanis ${ }^{\text {iiii }}$; Villareal Sánchez, Veronica ${ }^{\text {iv }}$.

La atención al cliente, el servicio, el producto y el precio como variables determinantes de la satisfacción del cliente en una pyme de servicios

Revista GEON, Vol. 6, No. 2, 2019

Pág. 18-24

Disponible en: $\underline{\text { https://doi.org/10.22579/23463910.159 }}$

\footnotetext{
i http://orcid.org/0000-0003-2302-6804

ii $\mathrm{http} / / /$ orcid.org/0000-0002-7097-9483

iii https://orcid.org/0000-0003-2286-2843

${ }^{i v}$ http://orcid.org/0000-0003-3370-2220
}

Esta publicación se encuentra bajo licencia: Creative Commons ReconocimientoNoComercialSinObraDerivada 4.0 Internacional

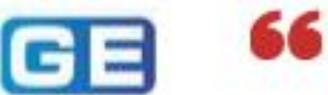

En la medida que logre satisfacer a sus clientes ofreciéndoles cumplir con los requerimientos que ellos desean podrá obtener la lealtad de ellos y poder así consolidar su nicho en el mercado de los servicios 99 


\section{La atención al cliente, el servicio, el producto y el precio como variables determinantes de la satisfacción del cliente en una pyme de servicios}

Customer service, service, product and price as determining variables of customer satisfaction in a service SME.

\section{Nemecio Lorenzo Valenzuela Salazar ${ }^{1}$, Clara Patricia Buentello Martínez ${ }^{2}$, Lilia Alanís Gómez ${ }^{3}$, Verónica L. Villarreal Sánchez ${ }^{4}$}

\begin{abstract}
Cómo citar este artículo / To reference this article:
\end{abstract}

Artículo de investigación:

Fecha de recepción: 2019/05/15

Fecha de aceptación: $2019 / 06 / 30$

Esta publicación se encuentra bajo licencia: Creative Commons ReconocimientoNoComercialSinObraDerivada 4.0 Internacional.

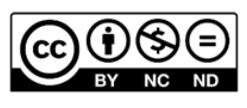

\begin{abstract}
Valenzuela Salazar, N., Buentello Martínez, C., Gómez, L., \& Villareal Sánchez, V. (2019). La atención al cliente, el servicio, el producto y el precio como variables determinantes de la satisfacción del cliente en una pyme de servicios. Revista GEON (Gestión, Organizaciones $Y$

Negocios), 6(2), 18-25. Recuperado a partir de http://revistageon.unillanos.edu.co/index.php/geon/article/view/159
\end{abstract}

\section{Resumen}

La calidad en el servicio se ha adquirido de mucha importancia en los últimos años en todos los negocios, debido a que los clientes actualmente tienen expectativas muy elevadas y las competencias son mayores, ya que cada empresa trata de igualarse o exceder dichas expectativas. Se deben ofrecer buenos servicios de calidad y anticiparse para responder a una amplia de necesidades de los clientes. La calidad en el servicio no solamente se trata de cómo saber manejar un producto, si no como servir a los clientes antes y después de su manejo. Es por eso por lo que la calidad en el servicio es utilizada en las organizaciones como dicha herramienta de diferenciación.

Palabras claves: Calidad en servicio, Satisfacción, Cliente, Negocios.

\footnotetext{
${ }^{1}$ Universidad Autónoma de Coahuila, Facultad de Administración y Contaduría, Miembro del CA en consolidación Ciencias Contables Administrativas, México, nlvs20000@yahoo.com.mx http://orcid.org/0000-0003-2302-6804

${ }^{2}$ Universidad Autónoma de Coahuila, Facultad de Administración y Contaduría, Responsable del CA en consolidación Ciencias Contables Administrativas, México, cbuente@uadec.edu.mx http://orcid.org/0000-0002-7097-9483

${ }^{3}$ Universidad Autónoma de Coahuila, Facultad de Administración y Contaduría, Miembro del CA en consolidación Ciencias Contables Administrativas, México, liliaalanisgómez@uadec.edu.mx http://orcid.org/0000-0002-5045-6140

${ }^{4}$ Universidad Autónoma de Coahuila, Facultad de Administración y Contaduría, Colaboradora del CA en consolidación Ciencias Contables Administrativas, México, veronicavillarreal@uadec.edu.mx http://orcid.org/0000-0003-3370-2220
} 


\begin{abstract}
Service quality has become very important in recent years in all businesses, because customers currently have very high expectations and skills are higher, as each company tries to match or exceed these expectations. Good quality services must be offered and anticipated to respond to a broad range of customer needs. Service quality is not only about how to manage a product, but how to serve customers before and after handling. That is why quality in the service is used in organizations as a tool for differentiation.
\end{abstract}

Keywords: Quality, Satisfaction, Customer, Client, Business

\section{Introducción}

Este estudio tiene como objetivo analizar la percepción que tienen los clientes preferenciales de una pyme de servicios con respecto a la satisfacción sobre los productos que compra el cliente en este tipo de negocio, para ello se consideraron las variables la atención al cliente, el servicio, el producto y el precio.

La investigación es de tipo no experimental descriptiva, siendo así que la investigación no experimental abarca todos los "estudios que realizan sin la manipulación deliberada de variables y en los que se observan los fenómenos en su ambiente natural después de analizarlo". (Hernández, Fernández \& Baptista, 2010).

\section{Marco Teórico}

Hoy en día hablar de la satisfacción del cliente es hablar de los deseos y expectativas de los consumidores. Cabe señalar que la satisfacción del cliente es muy importante en el ámbito de los negocios y que en la medida en que los clientes que consumen o adquieren productos, servicios o bienes estén satisfechos estos se convertirán en un actor preferencial de la compañía y probablemente volverá a usar sus servicios o productos, además de ayudar a promover dichos servicios a sus amistades, amigos y conocidos lo que significara un aumento en las ventas para la empresa. Para establecer una mejor comprensión de este estudio es importante definir las variables que se relacionan con esta investigación según algunos autores.

De acuerdo con (Blanco, 2001) la atención al cliente "Representa una herramienta estratégica que permite ofrecer un valor añadido a los clientes con respecto a la oferta que realicen los competidores y lograr la percepción de diferencias en la oferta global de la empresa".

Se puede decir que la atención al cliente es una acción encaminada a atraer aquellas personas o empresas para que consuman lo que los proveedores producen $\mathrm{u}$ ofrecen, $\mathrm{y}$ 
en consecuencia a este acto son ellos (clientes) los que valoran la atención que reciben.

Toda acción en la prestación del servicio debe estar dirigida a logar la satisfacción en el cliente. Esta satisfacción debe garantizarse en cantidad, calidad, tiempo y precio. (Solorzano Barrera, 2013)

Una sensación de placer o de decepción que resulta de comparar la experiencia del producto (o los resultados esperados) con las expectativas de beneficios previas. $\mathrm{Si}$ los resultados son inferiores a las expectativas, el cliente queda insatisfecho. Si los resultados superan las expectativas, el cliente queda muy satisfecho o encantado. (Kotler \& Armstrong, 2004).

Coral Sangri (2008).Define al servicio como: "El conjunto de actividades, beneficios o satisfactores que se ofrecen para su venta o se suministran con la venta.

Los servicios son actividades, beneficios o satisfacciones que se ofrecen en renta o a la venta, y que son esencialmente intangibles y no dan como resultado la propiedad de algo. (Sandhusen, 2002).

Un servicio se define de la siguiente manera: "Un servicio es una obra, una realización o un acto que es esencialmente intangible $y$ no resulta necesariamente cualquier cosa, favorable o desfavorable, que una persona recibe en intercambio Conjunto de atributos tangibles (forma, tamaño, color...) e intangibles (marca, imagen de empresa, servicio), que el comprador acepta, en un principio como algo que va a satisfacer sus necesidades. (Kotler Bloom \& Hayes, 2004)
El producto es el punto central de la oferta que realiza toda empresa u organización (ya sea lucrativa o no) a su mercado, meta para satisfacer sus necesidades y deseos, con la finalidad de lograr los objetivos que persigue. (Lamb Hair \& McDaniel, 2005).

Con respecto al Precio, este se puede definir como el monto de dinero que se paga por un producto o servicio. Kotler \& Armstrong,(2008), autores del libro Fundamentos de Marketing, el precio es en el sentido más estricto la cantidad de dinero que se cobra por un producto $\mathrm{o}$ servicio.

En términos más amplios, el precio es la suma de los valores que los consumidores dan a cambio de los beneficios de tener o usar el producto o servicio.

En el libro 199 Preguntas Sobre Marketing y Publicidad de Patricio Bonta y Mario Farber, ellos definen el precio como "la expresión de un valor. El valor de un producto depende de la imagen que percibe el consumidor. (Bonta P. \& Faber M. , 2003)

La Calidad, no podríamos iniciar este estudio sin considerar la importancia que tiene la calidad ya que este término según Álvarez representa un proceso de mejora continua, en el cual todas las áreas de la empresa buscan satisfacer las necesidades del cliente o anticiparse a ellas, participando activamente en el desarrollo de productos o en la prestación de servicios (Álvarez, 2006)

\section{Materiales y métodos}

Es el resultado de la indiferencia entre los estándares de comparación previos de los 
clientes y la percepción del rendimiento del servicio o bien de consumo. (Morales \& Hernández, 2004). Con una calidad superior a la de los competidores a través de un mejor precio, y con productos innovadores, se obtendrá un nicho de mercado lo que representará una clientela satisfecha y cautiva para el dueño del negocio.

\section{Objetivo: conla finalidad de conocer cual es la mediación de la calidad en la empresa Biemart se esta tealizando una encuesta la cual consiste de contestar las siguientes prequntas.}

\section{DATOS GENERALES}

EDAD 16a20ANOS Estado de prodencia 20 a 30 ANOS Frecuencia de compra 30 a 45 ANIOS Genero () FEMENNO (IMASCULINO 40 a50ANOS

INSTRUCCIONES: Porfavor conteste con una X larespuesta que usted considere mejor.

\begin{tabular}{ll}
\hline Excelente & 5 \\
\hline Bueno & 4 \\
\hline Regular & 3 \\
\hline Deficiente & 2 \\
\hline Muydeficiente & 1 \\
\hline
\end{tabular}

\section{ATENCION AL CLIENTE}

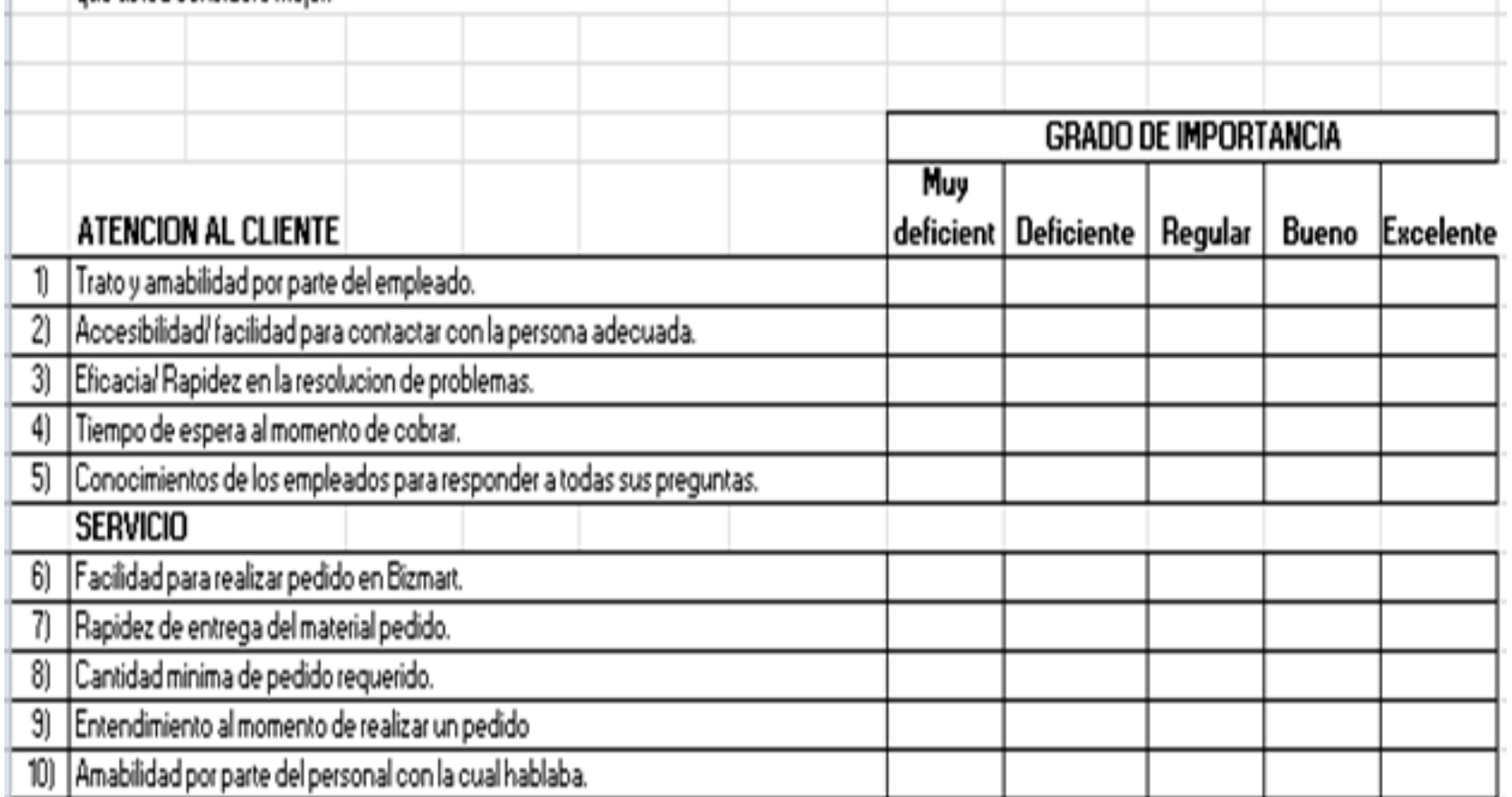
SERVICIO

6) Facilidad pararealizar pedido en Bizmatt.

7) Rapidez de entrega del material pedido.

8) Cantidadminima de pedido requerido.

9) Entendimiento al momento de realizar un pedido

10) Amabilidad por parte del personal con la cual hablaba.

Figura 1 formato de la encuesta que se utilizo Fuente: Elaboración propia

Según Méndez "los descriptivos identifican características del universo de investigación, señala formas de conducta y establece comportamientos concretos y

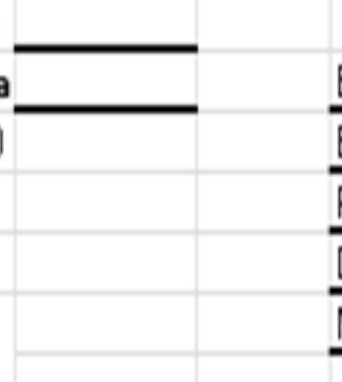

describe y comprueba asociación entre variables" (Méndez A., 1995)

La investigación de esta empresa Pyme está constituida solo por clientes 
preferenciales es decir aquellos que son los que más consumen o los más frecuentes, el número total de estos clientes es de 50 clientes.

Para obtener información se aplicó una encuesta de escala con cinco tipos de respuesta de tipo R. Likert, a los clientes de la empresa con el propósito de medir el nivel de su satisfacción, como lo muestra la figura siguiente.

Una vez aplicada la encuesta se utilizó el método de consistencia interna del Alfa de Cronbach, para determinar la confiabilidad.

Tabla \#1 Resultados de la prueba de Cronbach

\begin{tabular}{|c|c|c|c|}
\hline \multicolumn{2}{|c|}{ Escala : ANY } & & \\
\hline \multicolumn{4}{|c|}{ Resumen del proceso de casos } \\
\hline & & $N$ & $\%$ \\
\hline \multirow[t]{3}{*}{ Casos } & Válido & 15 & 100.00 \\
\hline & Excluido & 0 & 0.00 \\
\hline & Total & 15 & 100.00 \\
\hline \multicolumn{2}{|c|}{ Estadistica de fiabilidad } & & \\
\hline \multicolumn{2}{|c|}{ Alfa de Cronbach } & \multicolumn{2}{|c|}{ N. de Elementos } \\
\hline & 0.93 & & 20 \\
\hline
\end{tabular}

Fuente: Elaboración propia

Ya que como lo mencionan (Welch S, \& Comer J., 1988) La medida de la fiabilidad asume que los ítems (medidos en escala tipo Likert) miden un mismo constructo y que están altamente correlacionados. El resultado de la Prueba fue de 0,93 lo que nos indica que es muy confiable el instrumento como se observa en la tabla 1.

\section{Análisis de los resultados}

Por lo que respecta a las tres variables analizadas los resultados fueron las siguientes:
Atención al cliente. Esta variable está relacionada con preguntas como trato y amabilidad de los empleados, accesibilidad para encontrar a los empleados, el tiempo al momento de pagar el producto, el conocimiento que tiene los empleados sobre su trabajo. La percepción que tienen los encuestados son los siguientes: Solo el 1\% afirma que la atención que reciben es excelente, el $23 \%$ concuerdan que es bueno, el 54\% dicen que es regular y por último $22 \%$ es deficiente, y muy deficiente.

Por lo que respecta a los Servicios, las preguntas de esta variable son facilidad para realizar su compra o pedido, rapidez en la entrega de su pedido, servicio personalizado y la amabilidad del empleado al atenderle, la percepción que se observo fue: El $6 \%$ afirmo que es excelente, el $24 \%$ es bueno, el $48 \%$ que es regular y el $22 \%$ es deficiente, y muy deficiente.

Con respecto a la variable Productos la cual contiene preguntas como variedad del producto, relación de la calidad en el producto cumple con los requerimientos, calidad del producto mejor que la competencia, los resultados fueron: El 8\%, afirma que el producto es excelente, el $32 \%$ es bueno, el $42 \%$ dicen que es regular $16 \%$ es deficiente "caros", y por último el $2 \%$ que son muy caros.

Por último, la variable Precio que relaciona las preguntas la ubicación del precio, su accesibilidad ÿ variación, así como la competitividad de estos los resultados obtenidos fueron los siguientes: El $4 \%$ afirma que es precio es excelente, el $28 \%$ es bueno, el $42 \%$ dice que es regular 
$24 \%$ es caro, y por último el $2 \%$ son muy caro.

\section{Discusión}

Es indudable que en el sector servicios satisfacción es sinónimo de que tan buenos son los productos que oferta cualquier tipo de empresa a tal grado que esta es vista como una buena o una mala empresa y por ende esta valoración que el cliente hace de ellas repercutirá en sus ganancias.

Considerando entonces dicha importancia que tiene para los clientes la satisfacción, esta investigación está relacionada la percepción que tienen ellos con respecto a la atención y el servicio a la hora de adquirir un producto, el precio del producto que compran.

De ahí que, si se considera la atención al cliente, el servicio, el producto y el precio como variables determinantes de la satisfacción del cliente estas variables son percibidas de regular a buenos de acuerdo con los resultados obtenidos en la encuesta.

\section{Conclusiones}

Es indudable que este tipo de empresa tiene que esforzarme de acuerdo con los resultados de la percepción que tienen sus clientes preferenciales motivo por el cual deberá de esforzarse mucho más en el futuro si quiere mantenerse en el mercado, dado que hoy en día se vive una competencia agresiva en todos los negocios.
En la medida que logre satisfacer a sus clientes ofreciéndoles cumplir con los requerimientos que ellos desean podrá obtener la lealtad de ellos y poder así consolidar su nicho en el mercado de los servicios

\section{Bibliografía.}

Álvarez, M. (2006). Introducción a la Calidad: aproximación a los sistemas de gestión y herramientas de Calidad. España: Ed. Ideas Propias.

Blanco, P. (2001). Atención al Cliente. México: Pirámide.

Bonta P. \& Faber M. . (2003). Preguntas sobre Marketing y Publicidad. México: Norma.

Hernández, Fernández \& Baptista. (2010). Metodología de la Investigación. México: Mc Graw Hill.

Kotler \& Armstrong, G. (2004). Principles of Marketing. New Jersey: Pearson 10th edition.

Kotler Bloom \& Hayes, P. P. (2004). El Marketing de Servicios Profesionales. España: Paidós.

Kotler, P. (2012). Marketing. México: Pearson.

Lamb Hair \& McDaniel, C. (2005). Fundamentos de Marketing. Madrid. España: Paraninfo.

Méndez A., C. (1995). Metodología: Guía para elaborar diseños de investigación en ciencias económicas, contables y administrativas. México: McGraw Hill.

Morales \& Hernández. (12 de abril de 2004). La Calidad y su Gestión. Obtenido 
de

http://www.efdeportes.com/efd76/calidad .htm

Sandhusen, L. (2002). Mercadotecnia Internacional. México: Thompson.

Sangri Coral, A. (2008). Introducción a la Mercadotecnia. México: Patria.

Solorzano Barrera, G. J. (30 de Septiembre de 2013). Importancia de la calidad del servicio al cliente para el funcionamiento de las empresas. El Buzón de Pacioli(82), 25. Recuperado el 16 de noviembre de 2018, de http://www.itson.mx/publicaciones/paciol i/Documents/no82/Pacioli-82pdf

Welch S, \& Comer J. (1988). Quantitative Methods for Public Administration: Techniques and Applications. U.S.A: Books/ Cole Publishing Co. 